\title{
Anisotropic Spin Exchange in Pulsed Quantum Gates
}

\author{
N.E. Bonesteel and D. Stepanenko \\ Department of Physics and National High Magnetic Field Laboratory, Florida State University, Tallahassee, FL 32310 \\ D.P. DiVincenzo \\ IBM Research Division, T.J. Watson Research Center, Yorktown Heights, NY 10598
}

\begin{abstract}
We show how to eliminate the first-order effects of the spin-orbit interaction in the performance of a two-qubit quantum gate. Our procedure involves tailoring the time dependence of the coupling between neighboring spins. We derive an effective Hamiltonian which permits a systematic analysis of this tailoring. Time-symmetric pulsing of the coupling automatically eliminates several undesirable terms in this Hamiltonian. Well chosen pulse shapes can produce an effectively isotropic exchange gate, which can be used in universal quantum computation with appropriate coding.
\end{abstract}

PACS: 03.67.Lx, 71.70.Ej, 85.35.Be

The exchange interaction between spins is a promising physical resource for constructing two-qubit quantum gates in quantum computers [1] [n]. In the idealized case of vanishing spin-orbit coupling, this interaction is isotropic, and any Hamiltonian describing time-dependent exchange between two spin- $1 / 2$ qubits, $H_{0}(t)=J(t) \mathbf{S}_{1} \cdot \mathbf{S}_{2}$, commutes with itself at different times. Thus, the resulting quantum gate depends on $J(t)$ only through its time integral - a convenient simplification, particularly because, when carrying out quantum gates, the exchange interaction should be pulsed adiabatically on time scales longer than $\hbar / \Delta E$, where $\Delta E$ is a typical level spacing associated with the internal degrees of freedom of the qubits [3]. In addition, isotropic exchange alone has been shown to be sufficient for universal quantum computation, provided the logical qubits of the computer are properly encoded [6,7].

Given the potential advantages of isotropic exchange for quantum gates, it is important to understand the effect of the inevitable anisotropic corrections due to spinorbit coupling. When these corrections are included, the Hamiltonian describing time-dependent exchange is

$$
H(t)=J(t)\left(\mathbf{S}_{1} \cdot \mathbf{S}_{2}+\mathcal{A}(t)\right),
$$

where

$$
\mathcal{A}(t)=\boldsymbol{\beta}(t) \cdot\left(\mathbf{S}_{1} \times \mathbf{S}_{2}\right)+\mathbf{S}_{1} \cdot \mathbf{\Gamma}(t) \cdot \mathbf{S}_{2} .
$$

Here $\boldsymbol{\beta}(t)$ is the Dzyaloshinski-Moriya vector, which is first order in spin-orbit coupling, and $\boldsymbol{\Gamma}(t)$ is a symmetric tensor which is second order in spin-orbit coupling [8]. Although these corrections may be small, they will, in general, not be zero unless forbidden by symmetry. For example, Kavokin has recently estimated that $\beta(t)$ can be as large as 0.01 for coupled quantum dots in GaAs [9].

In this Letter we construct the quantum gates produced by pulsing $H(t)$. This is nontrivial because $H(t)$ typically does not commute with itself at different times. We represent the resulting gates using an effective Hamiltonian $\bar{H}(t)$, which we derive perturbatively in powers of the spin-orbit coupling. $\bar{H}(t)$ is simple to work with because it does commute with itself at different times. As an application of this effective Hamiltonian, we use it to tailor pulse forms that effectively eliminate any firstorder anisotropic corrections.

The quantum gate obtained by pulsing a particular $H(t)$ is found by solving the time-dependent Schrödinger equation $i \frac{d}{d t}|\Psi(t)\rangle=H(t)|\Psi(t)\rangle$ where $|\Psi(t)\rangle$ is the state vector describing the two spin-1/2 qubits (here, and in what follows, $\hbar=1$ ). In general this problem cannot be solved analytically. However, since we expect spin-orbit coupling to be small, it is natural to attempt a perturbative solution in powers of $\boldsymbol{\beta}(t)$ and $\boldsymbol{\Gamma}(t)$. To do this it is first necessary to solve the unperturbed $(\mathcal{A}(t)=0)$ problem exactly. This corresponds to pulsing the isotropic exchange interaction, for which the unitary time evolution operator at time $t$ is

$$
\begin{aligned}
U_{0}(t) & =T \exp \left(-i \int_{-\infty}^{t} J\left(t^{\prime}\right) \mathbf{S}_{1} \cdot \mathbf{S}_{2} d t^{\prime}\right) \\
& =\exp \left(-i x(t) \mathbf{S}_{1} \cdot \mathbf{S}_{2}\right),
\end{aligned}
$$

where

$$
x(t)=\int_{-\infty}^{t} J\left(t^{\prime}\right) d t^{\prime} .
$$

Here $T$ is the usual Dyson time ordering, and the second equality in (3) follows from the fact that isotropic exchange commutes with itself at different times. The unperturbed quantum gate produced by a full pulse is then $U_{0}(t \rightarrow \infty)=\exp \left(-i \lambda \mathbf{S}_{1} \cdot \mathbf{S}_{2}\right)$ where $\lambda=\int_{-\infty}^{\infty} J(t) d t$ is the pulse strength. This is a well studied class of quantum gates [1]. For $\lambda=\pi$ the result is a simple swap, and for $\lambda=\pi / 2$ it is a 'square root of swap' which, in conjunction with single-qubit rotations, can be used to construct a controlled-not gate [1].

We now consider the effect of the anisotropic corrections $\mathcal{A}(t)$. Given the evolution operator for the unperturbed system, it is possible to recast the problem 
in the interaction picture by introducing the state vector $\left|\Psi_{I}(t)\right\rangle \equiv U_{0}^{\dagger}(t)|\Psi(t)\rangle$ which satisfies the Schrödinger equation $i \frac{d}{d t}\left|\Psi_{I}(t)\right\rangle=J(t) \mathcal{A}_{I}(t)\left|\Psi_{I}(t)\right\rangle$ where $\mathcal{A}_{I}(t)=$ $U_{0}^{\dagger}(t) \mathcal{A}(t) U_{0}(t)$. A formal expression for the unitary operator describing a full pulse in this picture is then

$$
U_{I}=T \exp \left(-i \int_{-\infty}^{\infty} J(t) \mathcal{A}_{I}(t) d t\right) .
$$

Expanding the exponential in (5) generates the standard time-dependent perturbation theory expansion for $U_{I}$ in powers of $\mathcal{A}_{I}(t)$. Returning to the Schrödinger picture, the unitary operator describing the full quantum gate is $U=\exp \left(-i \lambda \mathbf{S}_{1} \cdot \mathbf{S}_{2}\right) U_{I}$.

Rather than simply carrying out the perturbation expansion for $U_{I}$, it is useful to parametrize the resulting quantum gate in terms of an effective Hamiltonian of the form

$$
\bar{H}(t)=J(t)\left(\mathbf{S}_{1} \cdot \mathbf{S}_{2}+\overline{\mathcal{A}}\right),
$$

where the time dependence of $J(t)$ is the same as in $H(t)$, and $\overline{\mathcal{A}}$ is independent of time. Unlike $H(t)$, the effective Hamiltonian $\bar{H}(t)$ commutes with itself at different times. Thus, after a full pulse, $\bar{H}(t)$ yields the quantum gate $\bar{U}=\exp \left(-i \lambda\left(\mathbf{S}_{1} \cdot \mathbf{S}_{2}+\overline{\mathcal{A}}\right)\right)$. Our goal is then to find the operator $\overline{\mathcal{A}}$ for which $\bar{U}$ is equal to the quantum gate produced by a full pulse of $H(t)$.

Because $H(t)$ is traceless at all times $t$, the corresponding unitary time evolution operator has determinant 1 , i.e., $U \in \mathrm{SU}[4]$. Requiring that our effective Hamiltonian produce the same quantum gate then implies that $\overline{\mathcal{A}}$ must also be a traceless Hermitian operator. The most general such operator acting on the Hilbert space of two qubits can be written

$$
\begin{aligned}
\overline{\mathcal{A}}=\overline{\boldsymbol{\beta}} \cdot\left(\mathbf{S}_{1} \times \mathbf{S}_{2}\right) & +\mathbf{S}_{1} \cdot \overline{\boldsymbol{\Gamma}} \cdot \mathbf{S}_{2} \\
& +\frac{\overline{\boldsymbol{\alpha}}}{2} \cdot\left(\mathbf{S}_{1}-\mathbf{S}_{2}\right)+\frac{\overline{\boldsymbol{\mu}}}{2} \cdot\left(\mathbf{S}_{1}+\mathbf{S}_{2}\right),
\end{aligned}
$$

where $\overline{\boldsymbol{\Gamma}}$ is a symmetric tensor. This can be seen by noting that $\overline{\mathcal{A}}$ is indeed traceless and Hermitian, and has 15 independent real valued parameters, the number of degrees of freedom for a $4 \times 4$ traceless Hermitian matrix.

Before proceeding it is instructive to classify the terms in $\overline{\mathcal{A}}$ according to their symmetry properties under inversion $\left(\mathbf{S}_{1} \leftrightarrow \mathbf{S}_{2}\right)$ and time reversal $\left(\mathbf{S}_{i} \rightarrow-\mathbf{S}_{i}\right)$. Under inversion $\overline{\boldsymbol{\beta}}$ and $\overline{\boldsymbol{\alpha}}$ change sign, while $\overline{\boldsymbol{\Gamma}}$ and $\overline{\boldsymbol{\mu}}$ do not. Since $\boldsymbol{\beta}(t)$ also changes sign under inversion this implies that $\overline{\boldsymbol{\beta}}$ and $\overline{\boldsymbol{\alpha}}$ are first order in spin-orbit coupling, while $\overline{\boldsymbol{\Gamma}}$ and $\overline{\boldsymbol{\mu}}$ are second order. Under time reversal $\overline{\boldsymbol{\alpha}}$ and $\overline{\boldsymbol{\mu}}$ change sign, while $\overline{\boldsymbol{\beta}}$ and $\overline{\boldsymbol{\Gamma}}$ are unaffected. We therefore expect that for time-reversal symmetric pulses, i.e., pulses for which $H\left(t_{0}-t\right)=H(t)$ (where $t_{0}$ is the center of the pulse), $\overline{\boldsymbol{\alpha}}$ and $\overline{\boldsymbol{\mu}}$ will vanish.

To determine $\overline{\mathcal{A}}$ for a given pulse we note that the requirement that $U=\bar{U}$ implies

$$
\begin{aligned}
T \exp \left(-i \int_{-\infty}^{\infty} J(t)\right. & \left.\mathcal{A}_{I}(t) d t\right) \\
& =T \exp \left(-i \int_{-\infty}^{\infty} J(t) \overline{\mathcal{A}}_{I}(t) d t\right),
\end{aligned}
$$

where $\overline{\mathcal{A}}_{I}(t)=U_{0}^{\dagger}(t) \overline{\mathcal{A}} U_{0}(t)$. Expanding both sides of (8) to a given order in spin-orbit coupling and equating matrix elements yields a set of 15 independent equations. These equations can then be solved for the parameters in $\overline{\mathcal{A}}$ in terms of $J(t), \boldsymbol{\beta}(t)$ and $\boldsymbol{\Gamma}(t)$.

We have carried out this calculation to obtain the following expressions valid to second order in spin-orbit coupling (i.e., second order in $\boldsymbol{\beta}(t)$ and first order in $\boldsymbol{\Gamma}(t)$ ),

$$
\begin{aligned}
& \overline{\boldsymbol{\alpha}}=\frac{1}{2 \sin (\lambda / 2)} \int_{-\infty}^{\infty} \boldsymbol{\beta}(t) \sin \left(x(t)-\frac{\lambda}{2}\right) J(t) d t, \\
& \overline{\boldsymbol{\beta}}=\frac{1}{2 \sin (\lambda / 2)} \int_{-\infty}^{\infty} \boldsymbol{\beta}(t) \cos \left(x(t)-\frac{\lambda}{2}\right) J(t) d t,
\end{aligned}
$$

$$
\overline{\boldsymbol{\mu}}=\frac{1}{4 \lambda} \int_{-\infty}^{\infty} J\left(t_{1}\right) d t_{1} \int_{-\infty}^{t_{1}} J\left(t_{2}\right) d t_{2}\left(\left(\boldsymbol{\beta}\left(t_{1}\right) \times \boldsymbol{\beta}\left(t_{2}\right)\right) \cos \left(x\left(t_{1}\right)-x\left(t_{2}\right)\right)+2(\overline{\boldsymbol{\alpha}} \times \overline{\boldsymbol{\beta}}) \sin \left(x\left(t_{1}\right)-x\left(t_{2}\right)\right)\right)
$$

and

$$
\overline{\boldsymbol{\Gamma}}_{a b}=\frac{1}{\lambda} \int_{-\infty}^{\infty} \mathbb{\Gamma}_{a b}(t) J(t) d t+\frac{1}{4 \lambda} \int_{-\infty}^{\infty} J\left(t_{1}\right) d t_{1} \int_{-\infty}^{t_{1}} J\left(t_{2}\right) d t_{2} I_{a b}\left(t_{1}, t_{2}\right) \sin \left(x\left(t_{1}\right)-x\left(t_{2}\right)\right),
$$

where

$$
I_{a b}\left(t_{1}, t_{2}\right)=2\left(\boldsymbol{\beta}\left(t_{1}\right) \cdot \boldsymbol{\beta}\left(t_{2}\right)-\bar{\beta}^{2}-\bar{\alpha}^{2}\right) \delta_{a b}-\left(\beta_{a}\left(t_{1}\right) \beta_{b}\left(t_{2}\right)+\beta_{a}\left(t_{2}\right) \beta_{b}\left(t_{1}\right)-2 \bar{\beta}_{a} \bar{\beta}_{b}-2 \bar{\alpha}_{a} \bar{\alpha}_{b}\right) .
$$

The criterion for the validity of these expressions is that $|\lambda \overline{\boldsymbol{\beta}}|,|\lambda \overline{\boldsymbol{\alpha}}| \ll 1$, where the factor of $\lambda$ is included because it is the product $\lambda \overline{\mathcal{A}}$ that enters the unitary op- erator $U$. It is then apparent that, for any finite $\boldsymbol{\beta}(t)$ and $\boldsymbol{\Gamma}(t)$, our expansion breaks down when $\lambda \rightarrow 2 \pi n$ for $n= \pm 1, \pm 2, \cdots$, because $\sin (\lambda / 2) \rightarrow 0$ at these points. 
However, for $\lambda \rightarrow 0$, while $\overline{\boldsymbol{\alpha}}$ and $\overline{\boldsymbol{\beta}}$ may diverge, $\lambda \overline{\boldsymbol{\alpha}}$ and $\lambda \overline{\boldsymbol{\beta}}$ will always remain finite, and so, provided $\boldsymbol{\beta}(t)$ and $\boldsymbol{\Gamma}(t)$ are small, our expansion remains valid in this limit [10].

As expected from symmetry considerations, we find that $\overline{\boldsymbol{\beta}}$ and $\overline{\boldsymbol{\alpha}}$ are first order in spin-orbit coupling, while $\overline{\boldsymbol{\Gamma}}$ and $\overline{\boldsymbol{\mu}}$ are second order. It is also readily verified that for a time-reversal symmetric pulse the integrals (9) and (11) for $\overline{\boldsymbol{\alpha}}$ and $\overline{\boldsymbol{\mu}}$ vanish. Thus these non time-reversal symmetric terms are only generated by pulses that are themselves not time-reversal symmetric.

Given the possibility of using the exchange interaction alone to perform universal quantum computation [6.], which depends crucially on the interaction being as close to isotropic as possible, a natural questions arises: Is it possible to ameliorate the effect of spin-orbit induced anisotropy on exchange-based quantum gates? We show below that the answer is yes - by carefully shaping pulses, it is possible to effectively eliminate the firstorder anisotropy terms leaving only a residual secondorder anisotropy.

There are two first-order terms in $\bar{H}(t), \overline{\boldsymbol{\alpha}}$ and $\overline{\boldsymbol{\beta}}$. We have already seen how to eliminate $\overline{\boldsymbol{\alpha}}$. By choosing a time-reversal symmetric pulse both $\overline{\boldsymbol{\alpha}}$ and $\overline{\boldsymbol{\mu}}$ will vanish from $\bar{H}(t)$. Although $\overline{\boldsymbol{\beta}}$ cannot similarly be eliminated, for appropriate pulse forms it can be effectively eliminated by performing a local rotation in spin space.

Let $\mathbf{S}_{2}^{\prime}=\mathbb{R} \cdot \mathbf{S}_{2}$ where $\mathbb{R}$ is a rotation matrix constructed to eliminate $\overline{\boldsymbol{\beta}}$ from $\bar{H}(t)$ so that

$$
\bar{H}(t)=J(t)\left(\mathbf{S}_{1} \cdot \mathbf{S}_{2}^{\prime}+\mathbf{S}_{1} \cdot \overline{\boldsymbol{\Gamma}}^{\prime} \cdot \mathbf{S}_{2}^{\prime}\right),
$$

where $\overline{\boldsymbol{\Gamma}}^{\prime}$ is a symmetric tensor. The precise form of this rotation depends on both $\overline{\boldsymbol{\beta}}$ and $\overline{\boldsymbol{\Gamma}}$ and cannot be expressed simply. However, up to second order in $\overline{\boldsymbol{\beta}}$, it is given by

$\mathbb{R}_{a b}=\delta_{a b}+\sum_{c} \epsilon_{a b c} \bar{\beta}_{c}-\left(\bar{\beta}^{2} \delta_{a b}-\bar{\beta}_{a} \bar{\beta}_{b}\right) / 2+O\left(\bar{\beta}^{3}\right)$,

and this is sufficient for our purpose of eliminating firstorder anisotropy. Using (15) one finds the residual anisotropy in (14) is, up to second order in $\overline{\boldsymbol{\beta}}$,

$$
\overline{\mathbb{\Gamma}}_{a b}^{\prime}=\overline{\mathbb{\Gamma}}_{a b}+\left(\bar{\beta}^{2} \delta_{a b}-\bar{\beta}_{a} \bar{\beta}_{b}\right) / 2+O\left(\bar{\beta}^{4}\right) .
$$

Thus, in this rotated coordinate system the first-order anisotropy vanishes and all corrections to the isotropic exchange interaction are second order in spin-orbit coupling.

The ability to eliminate $\overline{\boldsymbol{\beta}}$ from $\bar{H}(t)$ by simply rotating one qubit with respect to the other indicates a procedure for eliminating the first-order effects of spinorbit coupling in any quantum computer that uses tunable exchange for quantum gates. Suppose that symmetric pulses are used, so that $\overline{\boldsymbol{\alpha}}=0$, and pulse forms are chosen so that $\overline{\boldsymbol{\beta}}$ is the same for all pulse strengths $\lambda$. Then, if the qubits in the computer form a linear array, or any arrangement for which there are no closed loops of qubits connected by two-qubit gates, it will be possible to define a local spin-space coordinate system in which the effective interaction between any two neighboring qubits has the form (14). While this procedure does not completely eliminate the anisotropy, it does reduce it from an effect that is first order in spin-orbit coupling to one that is second order.

To demonstrate how (10) can be used to tailor pulse shapes that lead to the same $\overline{\boldsymbol{\beta}}$ for all pulse strengths $\lambda$, consider the family of pulses

$$
J(t ; \lambda)=J_{0}(\lambda) \operatorname{sech}^{2}(2 t / \tau(\lambda)),
$$

where $J_{0}(\lambda)$ and $\tau(\lambda)$ are, respectively, the pulse height and width, and the pulse strength is $\lambda=\int_{-\infty}^{\infty} J(t ; \lambda)=$ $J_{0}(\lambda) \tau(\lambda)$. To evaluate (10) it is also necessary to know the time dependence of $\boldsymbol{\beta}(t)$. Determining the precise form of this dependence will require a detailed microscopic study of the specific realization of the exchange interaction being considered. Here we take, as the simplest possible illustrative model, a linear dependence on $J(t ; \lambda)$,

$$
\boldsymbol{\beta}(t)=\boldsymbol{\beta}_{1} J(t ; \lambda),
$$

for which the integral (10) can be performed analytically, with the result

$$
\overline{\boldsymbol{\beta}}=\boldsymbol{\beta}_{1} \frac{4 J_{0}(\lambda)}{\lambda^{2}}(2-\lambda \cot (\lambda / 2)) .
$$

Also, because these pulses are time-reversal symmetric, (9) gives $\overline{\boldsymbol{\alpha}}=0$.

Equation (19) can be used to exploit the freedom to choose $J_{0}(\lambda)$ and $\tau(\lambda)$, while keeping $J_{0}(\lambda) \tau(\lambda)=\lambda$, to shape pulses that keep $\overline{\boldsymbol{\beta}}$ fixed for different pulse strengths. For example, if the pulse parameters for $\lambda=\pi$ (swap) are fixed to be $J_{0}(\pi)$ and $\tau(\pi)$, then, for general $\lambda$, one should take

$$
J_{0}(\lambda)=J_{0}(\pi) \frac{2 \lambda^{2}}{\pi^{2}} \frac{1}{2-\lambda \cot (\lambda / 2)},
$$

and

$$
\tau(\lambda)=\tau(\pi) \frac{\pi}{2 \lambda}(2-\lambda \cot (\lambda / 2)) .
$$

These pulse forms are shown in Fig. 1 for various values of pulse strength $\lambda$. Note that as $\lambda$ increases, the pulse height decreases. This is because $\overline{\boldsymbol{\beta}}$ becomes increasingly sensitive to $\boldsymbol{\beta}(t)$ with increasing $\lambda$ until, in the limit $\lambda \rightarrow 2 \pi$, the pulse height must go to zero if $\overline{\boldsymbol{\beta}}$ is to be kept constant. Although our perturbation expansion for $\overline{\mathcal{A}}$ breaks down as $\lambda \rightarrow 2 \pi$, for this example the pulse heights are chosen so that the parameters in $\overline{\mathcal{A}}$ remain small, and we are always within the perturbative 
regime. The pulse forms defined by (20) and 211) are therefore valid, even in this singular limit. Of course, in practice, pulses near $\lambda=2 \pi$ will be problematic because of the diverging pulse length.

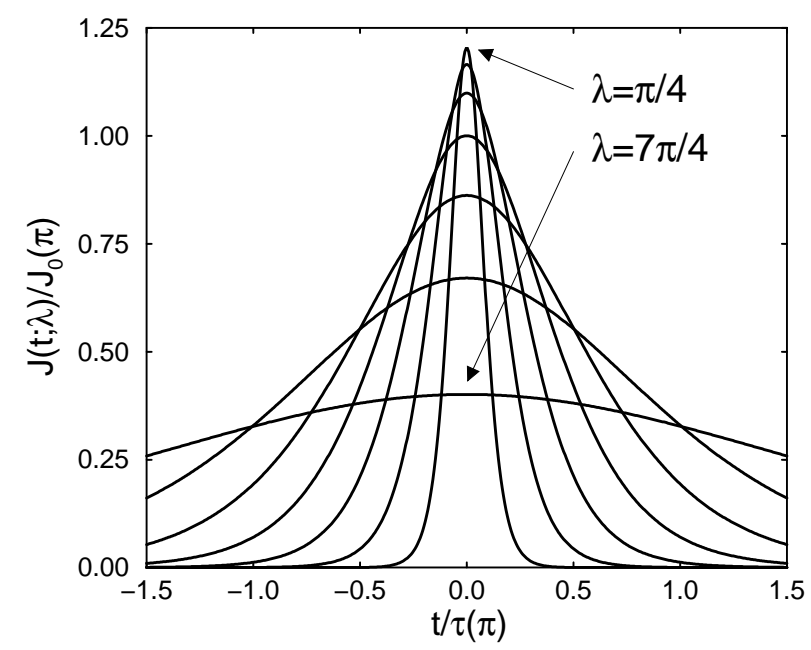

FIG. 1. Pulse forms tailored to produce the same $\bar{\beta}$ for different pulse strengths $\lambda$ for the example described in the text. Of the pulses shown, the narrowest with the highest peak is for $\lambda=\pi / 4$. $\lambda$ then increases in increments of $\pi / 4$ as the peak height decreases until, for the widest pulse with the lowest peak, $\lambda=7 \pi / 4$. As $\lambda \rightarrow 2 \pi$ the pulse height goes to zero.

Once the first-order corrections to $\bar{H}(t)$ are eliminated, the residual second-order anisotropy can be found by first evaluating (12) and then performing the local rotation to eliminate $\overline{\boldsymbol{\beta}}$. As a specific example, consider the special case for which the form of the pulsed Hamiltonian is

$$
H(t)=J(t) \mathbf{S}_{1} \cdot \mathbf{R}(t) \cdot \mathbf{S}_{2},
$$

where $\mathbf{I R}(t)$ is a time-dependent rotation matrix. Such rotated exchange is, in fact, precisely the form of anisotropy found microscopically when spin-orbit corrections are included in the usual Hubbard model treatment of superexchange 11, 12]. It has also been suggested that this form is appropriate for localized electrons in semiconductors [9]. In the present context (22) is of interest because if the rotation matrix $\mathbf{I R}(t)$ were independent of time, our local rotation scheme would eliminate anisotropy to all orders, rather than just to first order in spin-orbit coupling. It is therefore natural to ask to what degree the fact that $\mathbf{R}(t)$ depends on time spoils this hidden symmetry.

For the particular form of anisotropic exchange in (22), the symmetric anisotropy term is, to second order in $\boldsymbol{\beta}(t)$,

$$
\mathbb{\Gamma}_{a b}(t)=-\left(\beta(t)^{2} \delta_{a b}-\beta_{a}(t) \beta_{b}(t)\right) / 2+O\left(\beta(t)^{4}\right) .
$$

For this $\boldsymbol{\Gamma}(t)$, if we continue to take the pulse form (17) and $\boldsymbol{\beta}(t)$ from (18) then the expression (12) can be evaluated analytically. After performing the local rotation to eliminate $\overline{\boldsymbol{\beta}}$ we find, using (16), that the residual anisotropy in $\bar{H}(t)$ is

$$
\begin{aligned}
& \overline{\boldsymbol{\Gamma}}_{a b}^{\prime}=\frac{8 J_{0}(\lambda)^{2}}{3 \lambda^{4}}\left(\lambda^{2}+\right.6 \lambda \cot (\lambda / 2)-12) \\
& \times\left(\beta_{1}^{2} \delta_{a b}-\beta_{1_{a}} \beta_{1 b}\right)+O\left(\beta_{1}^{4}\right) .
\end{aligned}
$$

Thus even for the rotated exchange (22), if the rotation depends on time we are still left with residual secondorder anisotropy after a pulse.

To summarize, we have studied the effects of anisotropic corrections due to spin-orbit coupling on quantum gates produced by pulsing the exchange interaction between two spin-1/2 qubits. These quantum gates are parametrized by an effective Hamiltonian that commutes with itself at different times and produces the same quantum gate as a given pulse. Expressions for the various parameters in this effective Hamiltonian are obtained perturbatively in powers of spin-orbit coupling and used to shape pulses that effectively eliminate first-order spinorbit corrections to quantum gates. The ability to reduce spin-orbit effects from first order to second order should be useful for any quantum computing scheme which relies on isotropic exchange.

N.E.B. and D.S. acknowledge support from U.S. DOE Grant No. DE-FG02-97ER45639 and the NSF. D.P.D. is grateful for support from the National Security Agency and the Advanced Research and Development Activity through Army Research Office contract DAAG55-98-C0041.

[1] D. Loss and D.P. DiVincenzo, Phys. Rev. A 57, 120 (1998).

[2] B.E. Kane, Nature 393, 133 (1998).

[3] G. Burkard, D. Loss, and D.P. DiVincenzo, Phys. Rev. B 59, 2070 (1999).

[4] X. Hu and S. Das Sarma, Phys. Rev. A 61, 062301 (2000).

[5] R. Vrijen et al., Phys. Rev. A 62, 012306 (2000).

[6] D. Bacon et al., Phys. Rev. Lett. 85, 1758 (2000).

[7] D.P. DiVincenzo et al., Nature 408, 339 (2000).

[8] I. Dzyaloshinski, J. Phys. Chem. Solids 4, 241 (1958); T. Moriya, Phys. Rev. 120, 91 (1960).

[9] K.V. Kavokin, cond-mat/0011340.

[10] $\bar{\alpha}$ and $\bar{\beta}$ will only diverge as $\lambda \rightarrow 0$ if $J(t)$ changes sign. If $J(t)$ is always positive (or always negative) our expressions for the parameters in $\overline{\mathcal{A}}$ can be simplified by changing integration variables from $t$ to $x=x(t)$ and using the fact that $d x=J(t) d t$. It is then easily seen that $\bar{\alpha}$ and $\bar{\beta}$ remain finite as $\lambda \rightarrow 0$ for these pulses.

[11] L. Shekhtman, O. Entin-Wohlman, and A. Aharony, Phys. Rev. Lett. 69, 836 (1992).

[12] N.E. Bonesteel, Phys. Rev. B 49, 6088 (1994). 